\title{
Improvement in Clinical Outcomes, Physical Function, and Bodily Pain Following a 12 Week Course of Intermittent Pneumatic Compression Therapy in Patients with Chronic Venous Ulcers: Results of an Observational Longitudinal Retrospective Study
}

\author{
Eric Lullove ${ }^{1}$, Oscar M. Alvarez ${ }^{2}$ \\ $\square$ Corresponding author: Oscar M. Alvarez, oalvarez@calvaryhospital.org \\ Disclosures can be found in Additional Information at the end of the article
}

1. West Boca Medical Center, Boca Raton, FL 2. University Hospital, Newark Nj, Rutgers New Jersey Medical School

\begin{abstract}
Clinical records of 94 chronic venous ulcer patients (treated at two independent specialty centers) were included in a retrospective analysis to evaluate the effects of standard of care, plus continued intermittent pneumatic compression (IPC) therapy during and after wound closure.

Both clinical centers employed the Venous Clinical Severity Score (VCSS) to monitor clinical outcomes. IPC application varied slightly between the two clinical sites. One wound care center in South Florida treated patients $(\mathrm{n}=60)$ using a four chamber gradient IPC at $50 \mathrm{mmHg}$ for 45 minutes BID (five days on and two days off). The other center in New York treated patients ( $n=34)$ with a four chamber gradient IPC at 55 $\mathrm{mmHg}$ for one hour BID. Both wound care centers applied IPC therapy using standard adjustable $(95.3 \mathrm{~cm})$ half-leg sleeves. Both centers used the same IPC therapy pump (Bio Compression Model 2004, Bio Compression Systems Inc., Moonachie, NJ). All patients were seen weekly for standard evaluations and reapplication of compression bandages. Patient record analysis was for 12 consecutive weeks beginning at baseline (prior to IPC application). VCSS scores were recorded at monthly intervals.
\end{abstract}

Received 01/16/2014 Review began 01/22/2014 Review ended 03/31/2014 Published 04/28/2014

(c) Copyright 2014

Lullove et al. This is an open access article distributed under the terms of the Creative Commons Attribution License CC-BY 3.0., which permits unrestricted use, distribution, and reproduction in any medium, provided the original author and source are credited.

The incidence of ulcer healing was $80 \%$ after 12 weeks of IPC therapy. Symptomatic improvement was noted in every VCSS parameter measured. In the category of pain, there was a significant difference in the number of patients reporting severe pain before and after IPC therapy ( 42 at baseline vs. 0 after 12 weeks of IPC $[\mathrm{p}=0.004])$. Also, the number of patients reporting no pain before and after IPC therapy increased by $67 \%$ $(p<0.001)$. In the category of edema, significant improvement was noted after 12 weeks of IPC therapy in patients that had severe edema at baseline $(\mathrm{p}=0.017)$ and also in the number of patients where the edema resolved $(\mathrm{p}=0.004)$. Severe inflammation was significantly reduced in all study patients $(\mathrm{p}=0.022)$ and completely resolved in 60 of the 94 patients $(63.8 \%$, $\mathrm{p}<0.001)$.

The incidence of ulcer healing was $80 \%$ after 12 weeks of IPC therapy. Significant symptomatic improvement was noted in every VCSS parameter.

Categories: Dermatology, Pain Management, Physical Medicine \& Rehabilitation

Keywords: chronic venous insufficiency, venous ulcers, wound pain, edema, lymphedema, intermittent pneumatic compression, wound healing, venous clinical severity score, clinical severity score

\section{Introduction}

Venous leg ulcers (VLU) are chronic wounds that develop as a result of long-standing venous hypertension and chronic venous insufficiency (CVI) [1-4]. Venous ulcers account for 80 percent of lower extremity ulcerations [5]. Less common etiologies for lower extremity ulcerations include arterial insufficiency, prolonged pressure, diabetic neuropathy, and systemic illness, such as rheumatoid arthritis, vasculitis, osteomyelitis, and skin malignancy [2]. The overall prevalence of venous ulcers in the United States is approximately 1 percent [5]. Venous ulcers are more common in women and older persons [2,5]. The primary risk factors are older age, obesity, previous leg injuries, deep venous thrombosis, and phlebitis [6]. Venous ulcers usually recur and can persist from weeks to many years [7]. Severe complications include cellulitis, severe dermatitis, wound pain, osteomyelitis, and malignant change [8]. Although the overall prevalence is relatively low, the refractory nature of these ulcers increase the risk of morbidity and mortality, and have a

\section{How to cite this article}

Lullove E, Alvarez O M. (April 28, 2014) Improvement in Clinical Outcomes, Physical Function, and Bodily Pain Following a 12 Week Course of Intermittent Pneumatic Compression Therapy in Patients with Chronic Venous Ulcers: Results of an Observational Longitudinal Retrospective Study . Cureus 6(4): e175. DOI 
significant impact on patient quality of life [5, 7]. The financial burden of venous ulcers is estimated to be $\$ 2$ billion per year in the United States [9].

It is generally agreed upon that the cornerstone of treatment for CVI and VLU is compression. Compression increases the interstitial fluid pressure assisting the transport of fluid back into circulation, thereby reducing venous hypertension [10]. It is often thought that venous ulcers heal easily with standard graded compression consisting of short stretch and elastic bandages that provide 20-30 $\mathrm{mmHg}$ compression at the ankle. But data from randomized clinical trials have shown that less than $50 \%$ of patients with this type of compression healed within a 24 week period [11]. Greater incidence of wound closure has been demonstrated when compression is greater $(30-40 \mathrm{mmHg}$ ) [8]. The degree of compression for venous ulcer healing depends on the size of the leg, anatomical location of the ulcer, size and duration of the wound, degree of ambulation, severity of CVI, and availability of arterial blood supply (wound perfusion).

IPC therapy has been employed in the medical management of edema since 1955 [12]. The early IPC pumps employed a single chamber inflation cuff that applied compression to the entire extremity. Subsequent developments in IPC-involved pumps and sleeves with multiple chambers that delivered uniform, sustained segmental compression in a gradient fashion (with pressure in the distal chambers being greater than in the proximal chambers) enabling a sequential mechanism of distal to proximal delivery of compression [13-15]. IPC has been shown to accelerate the healing of venous ulcers and alleviate the symptoms of CVI in several clinical studies [16-19]. However, following a systematic review of IPC treatment for CVI and VLU [20], The Centers for Medicare and Medicaid Services (CMS) concluded that the body of evidence for IPC was not robust enough to merit reimbursement coverage across the board for the treatment VLU. Therefore, they issued a decision that IPC would only be covered for patients with refractory edema with significant ulceration of the lower extremities after a six month trial of standard therapies, such as compression bandages and stockings, had failed.

Our goal, in this observational retrospective review, was to measure and compare clinical outcomes and physical function in VLU patients who were treated with IPC therapy (according to CMS coverage guidelines). This review involves VLU patients from two independent clinical centers who were treated with IPC and had outcomes measured using the same instrument (VCSS) as an evaluative tool to record changes in disease severity over time and in response to treatment.

\section{Materials And Methods Institutional Review Board}

The governing IRB (Calvary Hospital IRB) ruled that this retrospective review of existing patient records was exempt from IRB review in accordance with 45CFR46.101. The data collected was without identifiers or link to identifiers. Additionally, the records review was conducted by investigators who would normally have access to the records as part of the patient's routine clinical care.

\section{Study design and patient population}

This longitudinal, observational study recruited a sample of subjects between January of 2010 and April of 2013 from two community-based wound care centers (one an independent outpatient clinic in Boca Raton, FL [ $\mathrm{n}=60$ ], and the other a hospital-based outpatient clinic in Bronx, NY [N=34]). Sites were asked to identify subjects with chronic VLU (> six month history of non-healing) who were receiving treatment with standard of care consisting of static compression, plus IPC therapy. These two clinical sites were selected because both measured clinical outcomes for their CVI patients using the Venous Clinical Severity Scoring System (VCSS), and both sites prescribed IPC therapy to treat recalcitrant VLUs that did not heal with static compression alone.

The participating physician or site coordinator conducted a retrospective chart review to collect demographic information, time since CVI diagnosis, duration of ulceration, VCSS scores at baseline (before the start of IPC therapy), and after their 12-week follow-up visit. VCSS was designed to provide a method for serial assessment and to give additional weight to more severe manifestations of chronic venous disease [21]. Although the usefulness of the original VCSS has been clearly demonstrated [22], several areas of deficiency have also been noted [23-24]. The revised VCSS (used in this study) addresses certain ambiguity in clinical descriptors, while preserving its strengths of thorough yet simple symptom evaluation.

\section{Statistical analysis}

Summary statistics (means and standard deviations [SD] for continuous variables and frequency distribution for categorical variables) were used to describe the sample. To evaluate the association between degree of pain and other outcomes, we used Kuskal-Wallis for continuous outcomes and Chi-square or Fisher's Exact Tests for categorical outcomes [25]. Statistical significance was evaluated at the 0.05 level. All analyses were 


\section{Cureus}

performed using PC-SAS version 9.2 (SAS Institute Cary, NC).

\section{Results}

Ninety-four patients were selected as evaluable from 103 that were screened. Six patients failed screening because they had mixed (arterial and venous) disease and three because they discontinued IPC therapy before 12 weeks.

\section{Demographic and clinical characteristics}

Table 1 describes the demographic and clinical characteristics of the patient population at each clinical center and when combined.

\begin{tabular}{|c|c|c|c|}
\hline Variable & Clinical Site \#1 (n=60) & Clinical Site \#2 ( $\mathrm{n}=34)$ & Pooled Demographics \\
\hline Mean age (years) & 74.6 & 64.4 & 69.5 \\
\hline Sex (\% female) & 53.2 & 55 & 54.1 \\
\hline Ulcer duration (months, median and absolute deviation) & $7.55(0.950)$ & 12.03 (2.737) & $9.78(1.843)$ \\
\hline Baseline ulcer size (cm2) & 18.5 & 26.3 & 22.4 \\
\hline Non-ambulatory (\%) & 0 & 23.5 & 11.7 \\
\hline >1 Ulcer (\%) & 21.6 & 32.3 & 26.5 \\
\hline Mean BMI & 30.8 & 34.4 & 32.6 \\
\hline
\end{tabular}

TABLE 1: Demographics and clinical characteristics, overall and from each clinical center

The demographics were not statistically significantly different between sites with the exception of immobility. All of the study patients were ambulatory at the South Florida site, whereas there were eight wheelchair-dependent subjects at the New York Clinical Center. The mean age of the 94 study patients with chronic venous insufficiency and lower leg ulceration was 69.5 years overall. There were 51 females and 43 males, and the mean ulcer age (history of non-healing) was 9.8 months. Twenty-five patients $(26.5 \%$ ) had more than one ulcer at baseline, and the mean body mass index (BMI) was 32.6. The BMI is a number calculated from a person's weight and height. BMI is a fairly reliable indicator of body fatness for most people. BMI does not measure body fat directly, but research has shown that BMI correlates to direct measures of body fat [26]. The Center for Disease Control (CDC) defines obese weight status as a BMI of $>30$ [27]. Interestingly, nearly $40 \%$ of study patients had a BMI of $>30$ and nearly $15 \%$ had a BMI of $>40$.

\section{Venous clinical severity scores (VCSS)}

The VCSS scores include pain, edema, inflammation, induration, and active ulcers. Pain includes aching, heaviness, fatigue, soreness, and burning. Edema presumes venous origin, includes interstitial edema but excludes edema secondary to nephrotic syndrome. Inflammation includes erythema, stasis (venous) eczema, and dermatitis. Induration presumes venous origin of secondary skin and subcutaneous changes (ie, chronic edema with fibrosis, hypodermatitis). It also includes atrophie blanche and lipodermatosclerosis. Active ulcers includes the longest and largest venous ulcer. VCSS scores were recorded on all study patients at monthly intervals during the follow-up visits. Overall, only nine patients (9.5\%) missed a single monthly follow-up visit, only three (3.1\%) missed two consecutive follow-up visits, and none missed three or more. The pooled VCSS scores for study patients at baseline (prior to treatment with IPC) and at week-12 are presented in Table 2. 


\section{Cureus}

\begin{tabular}{|c|c|c|c|}
\hline Symptom & Baseline & 12-Weeks & P-Value* \\
\hline Pain Severe Moderate Mild None & $\begin{array}{l}42(44.6 \%) 17(18 \%) 22(23.5 \%) \\
13(13.8 \%)\end{array}$ & 08 (8.5\%) 10 (10.6\%) 76 (80.8\%) & $\begin{array}{l}0.0040 .0510 .59 \\
0.001\end{array}$ \\
\hline Edema Severe Moderate Mild None & $63(67 \%) 22(23.4 \%) 9(9.5 \%) 0$ & $\begin{array}{l}013(13.8 \%) 14(14.8 \%) 67 \\
(71.2 \%)\end{array}$ & $\begin{array}{l}0.070 .0820 .27 \\
0.004\end{array}$ \\
\hline Inflammation Severe Moderate Mild None & $\begin{array}{l}28(29.7 \%) 54(57.4 \%) 12(12.7 \%) \\
0\end{array}$ & $03(3.1 \%) 31(32.9) 60$ & $\begin{array}{l}0.0220 .0180 .16 \\
0.001\end{array}$ \\
\hline Induration Severe Moderate Mild None & $\begin{array}{l}13(13.8 \%) 38(40.4 \%) 43(45.7 \%) \\
0\end{array}$ & $\begin{array}{l}6(6.3 \%) 29(30.8 \%) 34(36.1 \%) \\
25(26.5 \%)\end{array}$ & $\begin{array}{l}0.1460 .290 .09 \\
0.041\end{array}$ \\
\hline $\begin{array}{l}\text { Active ulcers Severe }(\geq 3) \text { Moderate (2) Mild } \\
\text { (1) Healed }\end{array}$ & $5(5.3 \%) 16(17.0 \%) 73(77.6)$ & $02(2.1 \%) 17(18.0 \%) 75(80 \%)$ & $\begin{array}{l}0.0660 .0590 .037 \\
0.002\end{array}$ \\
\hline
\end{tabular}

TABLE 2: Pooled VCSS Scores at Baseline (prior to treatment with IPC) and Week-12

${ }^{*} \mathrm{p}$-value depicts the statistical comparison between baseline VCSS scores and VCSS scores at week-12

The incidence of ulcer healing was $80 \%$ after 12 weeks of IPC therapy. Symptomatic improvement was noted in every VCSS parameter measured. The number of patients reporting severe pain before IPC therapy decreased $44.6 \%$ following 12 weeks of IPC therapy ( $(\mathrm{p}=0.004)$. Equally impressive, the number of patients that reported no pain before IPC therapy increased by $67 \%$ after 12 weeks of IPC $(p<0.001)$. In the category of edema, significant improvement was noted after 12 weeks of IPC therapy in patients that had severe edema at baseline $(\mathrm{p}=0.017)$ and also in the number of patients where the edema resolved completely $(\mathrm{p}=0.004)$. Severe inflammation was significantly reduced in all study patients $(\mathrm{p}=0.022)$ and completely resolved in 60 of the 94 patients $(63.8 \%$, $\mathrm{p}<0.001)$. At baseline, all 94 patients had some degree of induration. After 12 weeks of IPC therapy, complete resolution of induration was observed in $26.5 \%$ (25/94, $\mathrm{p}=0.041$ ). Although, a reduction in the degree of induration (level of fibrosis) was noted in most patients, the differences between scores at baseline and after 12 weeks of IPC therapy were not statistically significant.

\section{Discussion}

The gold standard for the treatment for CVI and VLU is compression. Often compression therapies, such as multi-layer, short stretch compression bandages, or prescription-grade compression stockings, are not enough to heal recalcitrant venous ulcers that are large $(>20 \mathrm{~cm} 2)$, or have a long history of non-healing [28]. In addition, patients affected with acquired lymphedema and dermal fibrosis, or patients that have a previous history of DVT, do not respond to traditional compression bandaging [29]. In these patients, gradient, sequential IPC therapy at home should be considered as an adjunct to traditional compression garments [30].

In this longitudinal, observational, retrospective study, we found that $80 \%$ of our patients (all of whom had failed six months or more of traditional compression bandaging) healed after 12 weeks of IPC therapy. In this study, all patients received IPC therapy while wearing compression bandages or (if healed) compression stockings. In most other previous IPC studies, IPC therapy was provided without a compression garment. Also, previous studies differed in the way IPC therapy was provided. Some evaluated single chamber devices [16] while others studied sequential gradient intermittent compression [17-18]; also, the level of compression was different [29] as well as the frequency of use [16-17, 29]. In the three largest studies available to date (involving a total of 151 patients), IPC therapy was found to be beneficial when compared to static compression bandages [17, 19, 30]. In the study by Smith, et al. [17] involving 45 patients, both the IPC and control groups received compression stockings and were instructed to wear stockings during their IPC (pumping) sessions. One of the 24 patients in the control group had healed within 12 weeks compared with 10 of 22 patients in the IPC group ( $p=0.009$ ). In a recently concluded RCT involving 52 subjects [30] that were treated with multi-layer compression bandages, the group that received IPC therapy had significantly better healing than the group treated with static compression alone $(\mathrm{p}=0.031)$.

In a systematic review of IPC therapy for the treatment of CVI and VLUs, Berliner, et al. [20] concluded that the available data at that time (2003) could not be relied on to inform the optimal choice of compression therapy or optimal protocol for patients with CVI or VLUs. From this study, The Centers for Medicare and Medicaid Services (CMS) issued a decision that IPC therapy would only be covered for patients with 
refractory edema with significant ulceration of the lower extremities after a six month trial of standard therapies, such as compression stockings or bandages, had failed. This decision, combined with the increased acceptance and use of IPC therapy, has sparked new controversy about the quantity and quality of published evidence that prove efficacy [31]. The authors of this review published in 2011 concluded that IPC therapy may increase healing compared with no compression, but it was not clear whether it increases healing when added to treatment with compression bandages, or if it could be used instead of compression bandages. This 2011 Cochrane review did not include the RCT recently completed by Alvarez, et al. that involved 52 subjects with recalcitrant venous ulcers [30]. In this trial, IPC therapy was studied as an adjunct to static compression (with short stretch or multi-layer bandages) and administered while the subjects were wearing compression bandages. It also did not include the study by Zaleska, et al. [32] where it was shown that IPC therapy was more effective when the pressures and timing were increased to $>50 \mathrm{mmHg}$ and $>50$ seconds, respectively.

\section{Conclusions}

In this retrospective observational study IPC therapy, the incidence of ulcer healing was nearly $80 \%$ after 12 weeks of IPC therapy and significant symptomatic improvement was noted in every VCSS parameter measured.

\section{Additional Information \\ Disclosures}

Human subjects: All authors have confirmed that this study did not involve human participants or tissue. Animal subjects: All authors have confirmed that this study did not involve animal subjects or tissue. Conflicts of interest: In compliance with the ICMJE uniform disclosure form, all authors declare the following: Payment/services info: All authors have declared that no financial support was received from any organization for the submitted work. Financial relationships: All authors have declared that they have no financial relationships at present or within the previous three years with any organizations that might have an interest in the submitted work. Other relationships: All authors have declared that there are no other relationships or activities that could appear to have influenced the submitted work.

\section{References}

1. Bergan JJ, Schmid-Schönbein GW, Smith PD, Nicolaides AN, Boisseau MR, Eklof B: Chronic venous disease. N Engl J Med. 2006, 355:488-98.

2. Falanga V: Venous ulceration. Wounds. 1996, 8:102-108.

3. Moffatt CJ, Doherty DC, Franks DDC: Community based leg ulcer classification and its relationship to healing. Int Angiol. 2001, 20:291.

4. Guarnera G, Tinelli G, Abeni D, Di Pietro C, Sampogna F, Tabolli S: Pain and quality of life in patients with vascular leg ulcers: An Italian multicentre study. J Wound Care. 2007, 16:347-51.

5. Phillips TJ, Dover JS: Leg ulcers. J Am Acad Dermatol. 1991, 25:965-87.

6. Collins L, Seraj S: Diagnosis and treatment of venous ulcers . Am Fam Physician. 2010, 81:989-96.

7. Valencia IC, Falabella A, Kirsner RS, Eaglstein WH: Chronic venous insufficiency and venous leg ulceration . J Am Acad Dermatol. 2001, 44:401-21.

8. Scottish Intercollegiate Guidelines Network (SIGN): Management of chronic venous leg ulcers. A national clinical guideline. (SIGN publication; no. 120). Scottish Intercollegiate Guidelines Network (SIGN), Edinburgh (Scotland); 2010.

9. Phillips T, Stanton B, Provan A, Lew R: A study of the impact of leg ulcers on quality of life: Financial, social, and psychologic implications. J Am Acad Dermatol. 1994, 31:49-53.

10. Blair, SD Wright DI, Backhouse CM, Riddle E, McCollum CN: Sustained compression and healing of chronic venous ulcers. BMJ. 1988, 297:1159-61.

11. Falanga V, Margolis D, Alvarez OM, et al.: Rapid healing of venous ulcers and lack of clinical rejection with an allogeneic cultured human skin equivalent. Human Skin Equivalent Investigators Group. Arch Dermatol. 1998, 134:293-300.

12. Brush BE, Heldt TJ: A device for relief of lymphedema . JAMA. 1955, 158:34-35.

13. Nelson EA, Mani R, Thomas K, Vowden K: Intermittent pneumatic compression for treating venous leg ulcers. Cochrane Database Syst Rev. 2011, Feb 16:CD001899. 10.1002/14651858.CD001899.pub3

14. Adams KE, Rasmussen JC, Dane C, et al.: Direct evidence of lymphatic function improvement after advanced pneumatic compression device treatment of lymphedema. Biomed Opt Express. 2010, 1:114-125.

15. Szuba A, Cooke JP, Yonsuf S, et al.: Decongestive lymphatic therapy for patients with cancer-related or primary lymphedema. Am J Med. 2000, 109:296-300.

16. Rowland J: Intermittent pump versus compression bandages in the treatment of venous leg ulcers . Aust N Z J Surg. 2000, 70:110-3.

17. Smith PC, Sarin S, Scurr SJ: Sequential gradient pneumatic compression enhances venous ulcer healing: A randomized trial. Surgery. 1990, 108:871-875.

18. McCulloch JM, Marler KC, Neal MB, Phifer TJ: Intermittent pneumatic compression improves venous ulcer healing. Adv Wound Care. 1994, 7:22-4. 


\section{Cureus}

19. Schuller JJ, Maibenco T, Magerman J, Ware M, Montalvo J: Treatment of chronic venous ulcers using sequential gradient intermittent pneumatic compression. Phlebology. 1996, 11:111-116.

20. Berliner E, Ozbilgin B, Zarin DA: A systematic review of pneumatic compression for treatment of chronic venous insufficiency and venous ulcers. J Vasc Surg. 2003, 37:539-44.

21. Rutheford RB, Padberg FT, Comerota RJ, Kirsner RL, Meissner MH, Moneta GL: Venous severity scoring: An adjunct to venous outcome assessment. J Vasc Surg. 2000, 31:1307-12.

22. Kakkos SK, Rivera MA, Mastagas MI, et al.: Validation of the new venous severity scoring system in varicose vein surgery. J Vasc Surg. 2003, 38:224-8.

23. Gilbert JL, Perrin MR, Allaert FA: Clinical presentation and venous severity scoring of patients with extended deep axial venous reflux. J Vasc Surg. 2006, 44:588-94.

24. Ricci MA, Emmerich J, Callas PW, et al.: Evaluating chronic venous disease with a new venous severity scoring system. J Vasc Surg. 2003, 38:909-15.

25. Elliot AC, Woodward WA: Statistical Analysis Quick reference Guidebook: With SPSS Examples . Sage Publications Inc, Thousand Oaks CA ; 2007.

26. CDC Health Information, About BMI for Adults, Centers for Disease Control . (2008). http://www.cdc.gov/nccdphp/dnpa/healthyweight/assessing/bmi/index.htm.

27. Defining Overweight and Obesity. http://www.cdc.gov/obesity/adult/defining.html.

28. Margolis DJ, Allen-Taylor L, Hoffstad O, Berlin JA: The accuracy of venous leg ulcer prognostic models in a wound care system. Wound Repair Regen. 2004, 12:163-8.

29. International Society of Lymphology: The diagnosis and treatment of peripheral lymphedema: 2013 Consensus Document of the International Society of Lymphology. Lymphology. 2013, 46:1-11.

30. Alvarez OM, Wendelken M, Markowitz L, Parker R, Comfort C: Effectiveness of Intermittent Pneumatic Compression for the Treatment of Venous Ulcers in Subjects with Secondary (Acquired) Lymphedema. Vein. 2012, 5:32-34

http://www.veindirectory.org/magazine/article/effectiveness_of_intermittent_pneumatic_compression_for_the_treat

31. Nelson EA, Mani R, Thomas K, Vowden K: Intermittent pneumatic compression for treating venous leg ulcers. The Cochrane Collaboration. The Cochrane Library. 2011, http://summaries.cochrane.org/CD001899/intermittent-pneumatic-compression-for-treating-venous-legulcers.

32. Zaleska M, Olszewski WL, Jain P, Gogia S, Rekha A, Mishra S, Durlik M: Pressures and timing of intermittent pneumatic compression devices for efficient tissue fluid and lymph flow in limbs with lymphedema. Lymphat Res Biol. 2013, 11:227-32. 10.1089/1rb.2013.0016 